\title{
Stochastic Loewner Evolution Relates Anomalous Diffusion and Anisotropic Percolation
}

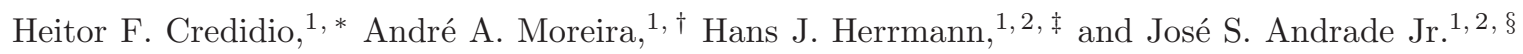 \\ ${ }^{1}$ Departamento de Física, Universidade Federal do Ceará, Campus Do Pici, 60455-760 Fortaleza, Brazil \\ ${ }^{2}$ Computational Physics IfB, ETH Zurich, Stefano-Franscini-Platz 3, CH-8093 Zurich, Switzerland
}

(Dated: August 30, 2018)

\begin{abstract}
We disclose the origin of anisotropic percolation perimeters in terms of the Stochastic Loewner Evolution (SLE) process. Precisely, our results from extensive numerical simulations indicate that the perimeters of multi-layered and directed percolation clusters at criticality are the scaling limits of the Loewner evolution of an anomalous Brownian motion, being subdiffusive and superdiffusive, respectively. The connection between anomalous diffusion and fractal anisotropy is further tested by using long-range power-law correlated time series (fractional Brownian motion) as driving functions in the evolution process. The fact that the resulting traces are distinctively anisotropic corroborates our hypothesis. Under the conceptual framework of SLE, our study therefore reveals new perspectives for mathematical and physical interpretations of non-Markovian processes in terms of anisotropic paths at criticality and vice-versa.
\end{abstract}

PACS numbers: 89.75.Da, 64.60.al, 05.40.Jc

The Stochastic Loewner Evolution (SLE) [1] has revolutionized our understanding of two dimensional loopless paths, as recognized among others by several Fields medals [2, 3]. It provides a mapping between these paths and a real valued function, called "driving function", that is a random walk if the path is a conformally invariant fractal. This establishes a relation between the fractal dimension of the path and the diffusion constant of the random walk. Although several generalizations have been proposed [4 7], due to its nature, SLE has been restricted to isotropic models 8 14]. However, anisotropic paths, namely, paths with a preferential direction, appear quite commonly in Physics. By numerically determining the driving function of anisotropic paths, we discover that they are consistently mapped onto correlated random walks, meaning that the Markovian property of the driving function is violated. More precisely, we show that resulting anomalous diffusion is characterized by an exponent that is related to the degree of anisotropy. This behavior can be subdiffusive, as it is the case for the hull of directed percolation or superdiffusive, as found for multi-layered percolation.

The chordal variety of SLE (the one we will focus on this work) deals with curves $\gamma_{t}$ that start at the origin and grow towards infinity while restricting themselves to the complex upper half-plane $\mathbb{H}$. The curve $\gamma_{t}$ is connected to a real-valued driving function $U_{t}$ through the relation $\gamma_{t}=g_{t}^{-1}\left(U_{t}\right)$ where $g_{t}(z)$ is the solution of Loewner's equation [19, 20],

$$
\partial_{t} g_{t}(z)=\frac{2}{g_{t}(z)-U_{t}}, \quad g_{0}(z)=z .
$$

In his seminal work [1], Schramm showed that if the measure over $\gamma_{t}$ displays conformal invariance and domain Markov property, then the only possibility is that $U_{t}$ be a Brownian motion with a single free parame- ter $\kappa$, the diffusion coefficient. This is often written as $U_{t}=\sqrt{\kappa} B_{t}$ where $B_{t}$ is a standard Brownian motion (with diffusion coefficient equal to unity). The value of $\kappa$ is related to the geometric properties of $\gamma_{t}$, including its fractal dimension, which is determined by the relation $d_{f}=\min \left(1+\frac{\kappa}{8}, 2\right)$ [21]. A few lattice models have been shown to converge to SLE in the continuum limit 1, 12, 22], and many more are conjectured to do so $9,13,14,23$ 25]. Of particular interest is the proof

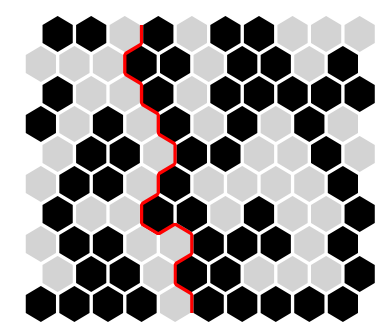

(a)

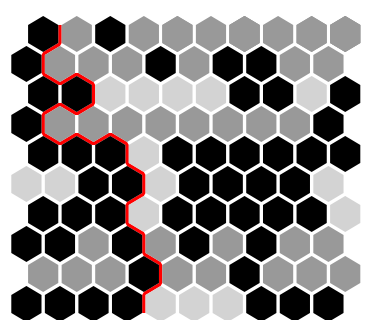

(b)

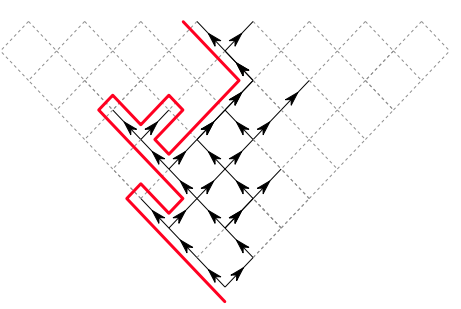

(c)

FIG. 1. Percolation models used to generate the SLE curves. (a) Regular percolation, where each site is occupied with the same probability $p$ [15]. (b) Multi-layered percolation, where some rows are occupied with probability $p+\Delta$ (dark gray rows) and others with $p-\Delta$ (light gray rows) [16]. (c) Directed percolation is a spreading process which starts at the bottom of the tilted lattice and can only advance upwards with probability $p$ [17]. 

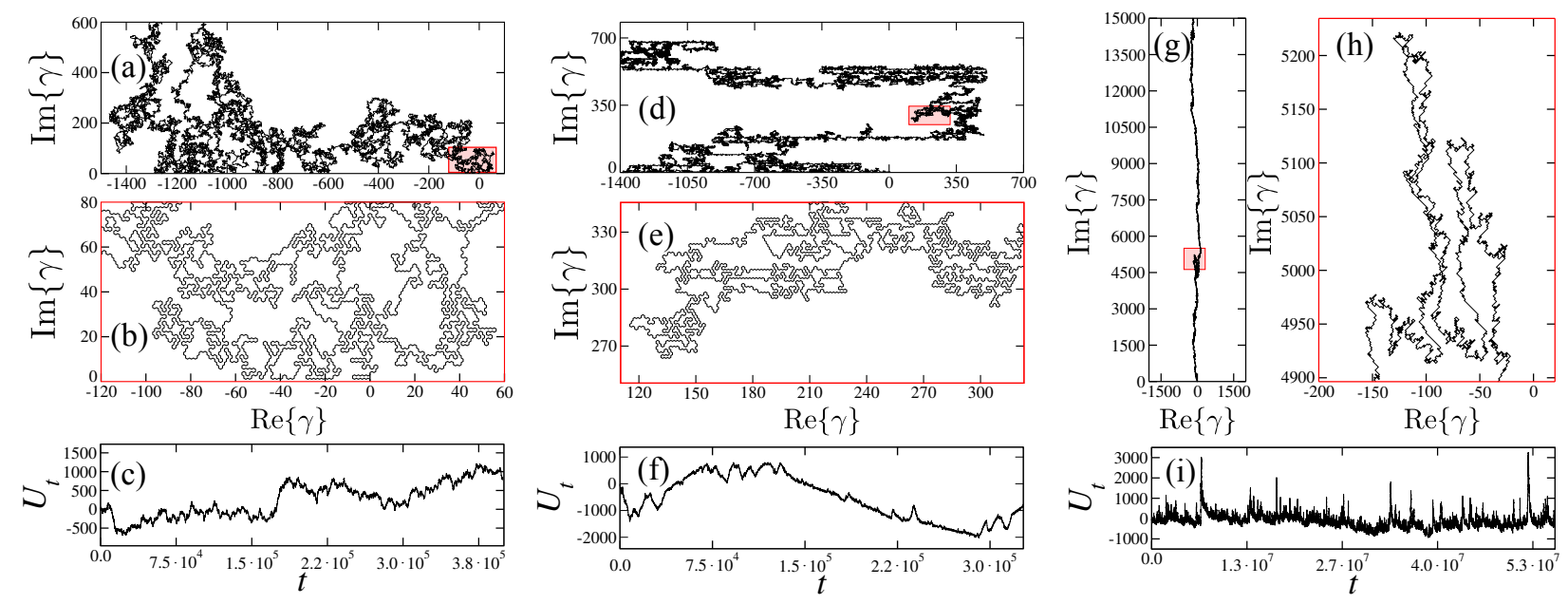

FIG. 2. Examples of cluster perimeters at the critical point of (a) isotropic percolation on a triangular lattice $\left(p_{c}=0.5\right)$, $(\mathrm{d})$ multi-layered percolation also on a triangular lattice $\left(\Delta=0.2\right.$ and $\left.p_{c}=0.5\right)$, and $(\mathrm{g})$ directed percolation on a square lattice $\left(p_{c} \approx 0.644\right)$. A detail of each curve in $(\mathrm{a}),(\mathrm{d})$ and $(\mathrm{g})$ (pink square) can be seen in (b), (e) and (h), respectively. The driving functions obtained by applying the zipper algorithm [18] to the curves (a), (d) and (g) are shown in (c), (f) and (i), respectively.

that the perimeter of a percolation cluster on a triangular lattice follows SLE with $\kappa=6$ as a scaling limit, allowing for a formal computation of its critical exponents [8].

In this work we explore the possibility of using Loewner evolutions to study anisotropic fractal systems, i.e., systems with different critical exponents in each direction. These systems are not scale invariant, therefore not conformally invariant either. We are particularly interested in two variants of the percolation model that show anisotropic behavior, namely, multi-layered percolation and directed percolation (see Fig. 11). Precisely, we generate the border of percolating clusters, numerically compute their corresponding driving function, and then analyze the diffusive properties of these numerical sequences. In the general case, we expect that the mean square displacement of $U_{t}$ behaves like,

$$
\left\langle U_{t}^{2}\right\rangle \rightarrow b t^{\alpha}
$$

as $t \rightarrow \infty$. In the case of traditional SLE, $\alpha=1$ and $b=\kappa$. The driving functions of anisotropic percolation models, we found, display very distinctive anomalous diffusive behavior $(\alpha \neq 1)$. Finally, we show that our approach is also valid in the opposite direction, namely, the SLE consistently leads anomalously diffusive driving functions to traces that display clear anisotropic scaling.

In order to evaluate the SLE driving function of the cluster perimeters we used the zipper algorithm with a vertical slit discretization [18, 26]. In this method, given a lattice curve $\left\{0, \gamma_{1}, \gamma_{2}, \ldots, \gamma_{N}\right\}$, its driving function can be recovered by applying the relations,

$$
t_{k}=\frac{1}{4} \sum_{j=1}^{k} \operatorname{Im}\left\{\omega_{j}\right\}^{2} \quad U_{t_{k}}=\sum_{j=1}^{k} \operatorname{Re}\left\{\omega_{j}\right\}
$$

where the $\omega_{k}$ 's are determined recursively by

$$
\omega_{k}=f_{k-1} \circ f_{k-2} \circ \ldots \circ f_{1}\left(\gamma_{k}\right) \quad \omega_{1}=\gamma_{1},
$$

and $f_{k}(z)$

$$
f_{k}(z)=i \sqrt{-\operatorname{Im}\left\{\omega_{k}\right\}^{2}-\left(z-\operatorname{Re}\left\{\omega_{k}\right\}\right)^{2}} .
$$

This algorithm, however, does not guarantee that the discretized times $t_{k}$ are equally distributed, even for curves of same length and step size. To obtain an ensemble of curves defined for the same time sequence, we linearly interpolate the obtained driving function at equally spaced points in logarithmic time in the interval $\left[1, \log t_{f}\right]$, for some suitable $t_{f}$.

As already mentioned, we also performed the opposite operation of computing the SLE trace from a given driving function The process is simply the inversion of the algorithm previously described. Given a discretized driving function $U_{t}=\left\{0, U_{t_{1}}, \ldots, U_{t_{N}}\right\}$, the trace can be obtained by repeatedly applying the functions

$$
\gamma_{i}=g_{0} \circ g_{1} \circ \ldots \circ g_{i}(0),
$$

where the mappings are also chosen to represent a vertical slit discretization,

$$
g_{i}(z)=i \sqrt{4\left(t_{i}-t_{i-1}\right)^{2}-z^{2}}+\left(U_{t_{i}}-U_{t_{i-1}}\right) .
$$

Instead of using an approximate algorithm [27], we chose to use the one described above, as they are exact (for a given discretization). Their complexity scales as $O\left(N^{2}\right)$ which can get quite time consuming, specially for large values of $\kappa$, requiring a large number of points to get accurate results. We resorted to GPU parallelization (where each $\gamma_{k}$ is computed by a single thread) to achieve satisfactory accuracy. 


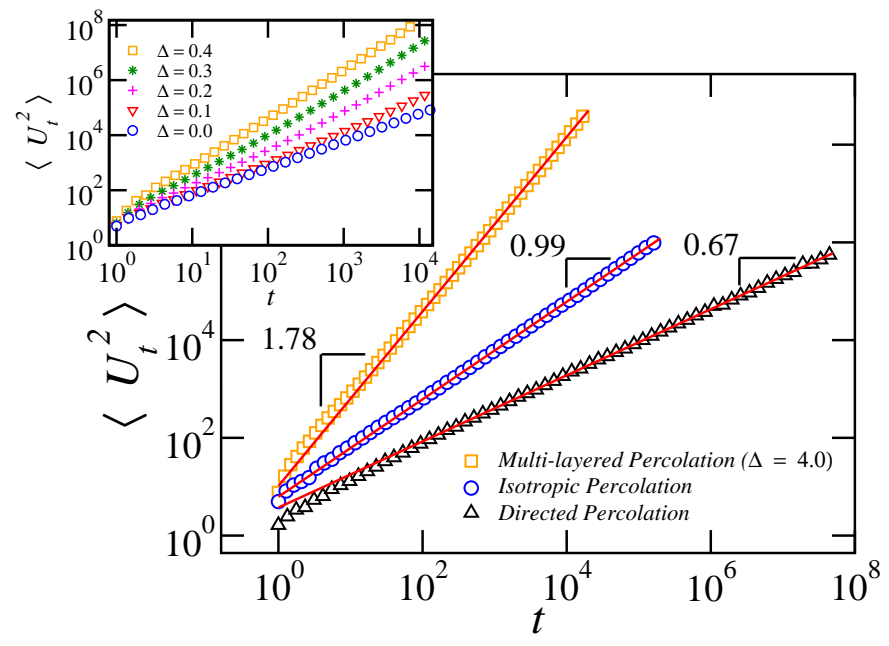

FIG. 3. Mean squared displacement of the driving functions for the three percolation models studied. The curves are the results of the numerical procedure described in the text applied to $10^{4}$ realizations of each type of percolation model. The 95\% confidence intervals were bootstrapped over 400 resamplings [28], but, being smaller than the symbols, are not shown. As expected, in the case of isotropic percolation, the displacement scales linearly with time, while it shows instead a distinctive subdiffusive behavior for directed percolation, with an exponent $\alpha \approx 0.67$. In the case of multi-layered percolation, a clear superdiffusive behavior, with an exponent $\alpha \approx 1.78$, can be observed for $\Delta=0.4$. The inset shows how this anomalous diffusion regime is gradually achieved as we increase the degree of anisotropy $\Delta$.

We start by testing our approach on standard isotropic percolation, which has been extensively studied as a simple but rather rich and illustrative model for criticality [15]. It is basically a lattice model with binary disorder, where each site (or bond) is occupied with probability $p$. For a given critical probability $p_{c}$, the presence of a giant spanning cluster is detected. In the thermodynamic limit, if $p<p_{c}$, where $p_{c}$ is the percolation threshold, the system never percolates, otherwise it always does. In particular, $p_{c}=1 / 2$ for site percolation on the triangular lattice [15]. It has been mathematically proven that the perimeter of the giant cluster at the critical point 29] follows SLE with $\kappa=6$ [8]. We perform simulations with $10^{4}$ realizations of percolation perimeters of length $10^{5}$ lattice units generated using the algorithm described in Ref. [30] on the triangular lattice. Fixed boundary conditions are adopted, in which every site on the left side of the bottom row is always unoccupied and the ones on the right side are always occupied. In Fig. 2 a we show a typical realization of an isotropic percolation perimeter and the corresponding driving function, as computed using the algorithm Eqs. 3.5. Finally, from the driving functions, we calculate their mean squared displacement $\left\langle U_{t}^{2}\right\rangle$ as a function of time, and find that $\kappa=6.27 \pm 0.30$ and $\alpha=0.996 \pm 0.005$, as shown in Fig. 3h.
Unlike regular percolation, where each site or bond is occupied with probability $p$, in multi-layered percolation this is done with probability $p \pm \Delta$, where $\Delta \in\left[0, \frac{1}{2}\right]$, and the signs plus or minus are chosen randomly with equal probabilities for each row of the lattice [16, 31]. Here, the parameter $\Delta$ represents the degree of anisotropy of the system, with $\Delta=0$ being equivalent to isotropic (regular) percolation.

We generate an ensemble of $10^{4}$ multi-layered percolation perimeters of length $10^{5}$ lattice units on a triangular lattice at the critical point for different values of $\Delta$. For every value of $\Delta$ we found $p_{c}=0.5$ using the cluster perimeter method [32]. As for the standard percolation case, after calculating the driving functions, an example of which is shown in Fig. 2b, we compute the corresponding mean square-displacement to find that it exhibits characteristic superdiffusive behavior for every value of $\Delta>0$. As can be observed in the inset of Fig. 3, however, a long transient behavior is present for small values of $\Delta$ before a distinctive power-law behavior is established. For $\Delta=0.4$, after a short transient, the leastsquares fit to the data gives a power-law, $\left\langle U_{t}^{2}\right\rangle=b t^{\alpha}$, with $b=10.38 \pm 0.68$ and $\alpha=1.78 \pm 0.01$, which extends over more than three orders of magnitude.

Next we investigate the diffusive behavior of driving functions generated from directed percolation perimeters. As defined, directed percolation is a spreading process where a cluster can only grow along preselected directions in a lattice, and each site is occupied with probability $p$ [17. Shown in Fig. 15 is a typical realization of a directed percolation perimeter generated on a tilted square lattice at the critical point, $p_{c}=0.644700185(5)$ [33]. Using this simulation setup, the perimeters of the spanning clusters are obtained here using a simple walker algorithm, as illustrated by the red curve in the example shown in Fig. 1c. From the ensemble of the generated driving functions, once more the resulting mean square displacement displays a characteristic anomalous behavior. Precisely, the least-squares fit to the data in the scaling region yields subdiffusive diffusion, as shown in Fig. 3, with a pre-factor $b=3.74 \pm 0.07$ and an exponent $\alpha=0.676 \pm 0.001$.

The results obtained by the previous analysis suggest that the presence of long-range correlations in the driving function should lead, through the Loewner evolution process, to anisotropic fractal traces, and vice-versa. In order to test this hypothesis, we analyze the behavior of traces driven by stochastic processes exhibiting anomalous diffusion. We choose to use fractional Brownian time series generated according to a given Hurst exponent $H$, which is related to the diffusion exponent by $\alpha=2 H[34]$.

We generated the drive $U_{t}$ as a fractional Brownian Motion with Hurst exponent $H$ and diffusion constant $b$ in $N$ time steps $t_{i}$ uniformly spaced in the interval $\left[0, t_{f}\right]$. In order to simulate fractional Brownian motions with reasonable control over the diffusive constant $b$, the 
Davies-Harte algorithm was used [35]. The $\gamma_{t_{i}}$ were computed from $U_{t_{i}}$ using Eq. 6. We then interpolated the trace $\gamma(\ell)$ (the same $\gamma_{t_{i}}$ as before, but parametrized by its length instead of the Loewner time) in $M$ equally spaced points $\ell_{i} \in\left[0, \ell_{\max }\right]$. This interpolation step was necessary because the zipper algorithm generates discretized traces with highly non-uniform step sizes $\left|\gamma_{i}-\gamma_{i-1}\right|$. Although this does not diminish the intrinsic error of the algorithm, it makes the analysis easier to perform. In order to study whether the scaling is isotropic or anisotropic, the root mean squared estimation of the displacement of the trace was computed in each direction, that is,

$$
F_{X}(i \Delta \ell)=\sqrt{\frac{1}{M-i} \sum_{j=0}^{M-i}\left[X\left(\ell_{j+i}\right)-X\left(\ell_{j}\right)\right]^{2}},
$$

where $X(\ell)=\operatorname{Re}\{\gamma(\ell)\}$. Analogously, $F_{Y}(i \Delta \ell)$ is defined taking instead $Y(\ell)=\operatorname{Im}\{\gamma(\ell)\}$.

Our numerical scheme was applied to three sets of times series, each with 100 realizations generated to reproduce the corresponding properties (in terms of $H$, $b$ and $t_{f}$ ) of the driving functions originated from the isotropic and anisotropic percolation traces previously investigated. More precisely, the first set of time series corresponds to uncorrelated Brownian motion, the second to correlated or persistent, and the third is anti-correlated or anti-persistent [36]. The remaining parameters $(N$, $M$ and $\left.\ell_{\max }\right)$ are chosen to ensure the accuracy of our results. The precise values of all parameters adopted in the simulations are reported in Table I. Figure 4 shows that the traces evolved from these time series have similar behavior to their corresponding percolation models, in the sense that a clear anisotropy can be observed in the correlated and anti-correlated simulations, while the uncorrelated one displays isotropic behavior, as expected.

In summary, our numerical analysis offers compelling evidence that a variation of the Stochastic Loewner Evolution, obtained by taking as driving function a stochastic process with anomalous diffusion, may be the scaling limit of anisotropic critical models. In particular we looked at two anisotropic variants of percolation: directed percolation and multi-layered percolation. The former was found to be associated with subdiffusive driving functions, while the latter are superdiffusive. We also tested the inverse relation, finding that driving functions with anomalous diffusion do indeed generate traces with anisotropic features. This possibility opens new questions, like how the critical exponents of SLE traces depend on the addition of long-term correlations to the driving function. Moreover it would be interesting to know if one can obtain exponents of actual physical models with such a generalized theory. We expect that the further developments of this new variant of SLE may provide some insight on the critical behavior of anisotropic systems, the same way the original SLE was to isotropic systems.

\begin{tabular}{|l||c|c|c|c|c|c|}
\hline & $H$ & $b$ & $t_{f}$ & $N$ & $M$ & $\ell_{\max }$ \\
\hline \hline Ensemble 1 & 0.5 & 6.0 & $2 \cdot 10^{5}$ & $10^{6}$ & $10^{5}$ & $2 \cdot 10^{4}$ \\
\hline Ensemble 2 & 0.8 & 16.0 & $3 \cdot 10^{4}$ & $10^{6}$ & $10^{5}$ & $8 \cdot 10^{4}$ \\
\hline Ensemble 3 & 0.33 & 3.8 & $5 \cdot 10^{7}$ & $10^{6}$ & $10^{5}$ & $2 \cdot 10^{4}$ \\
\hline
\end{tabular}

TABLE I. Simulation parameters used to generate the SLE traces. $H$ is the Hurst exponent and $b$ is the diffusion coefficient of the fractional Brownian motion used as driving function. The curves were computed for $N$ times $t_{i}$ equally spaced in the interval $\left[0, t_{f}\right]$. The resulting trace is reparametrized as a function of its length and interpolated in $M$ points equally spaced in the interval $\left[0, \ell_{\max }\right]$.

We thank the Brazilian agencies CNPq, CAPES, and FUNCAP, the National Institute of Science and Technology for Complex Systems in Brazil, and the European Research Council (ERC) Advanced Grant No. 319968FlowCCS for financial support.

\footnotetext{
* credidio@fisica.ufc.br

$\dagger$ auto@fisica.ufc.br

$\ddagger$ hans@fisica.ufc.br

$\S$ soares@fisica.ufc.br
}

[1] O. Schramm, Israel J. Math. 118, 221 (2000).

[2] D. Mackenzie, Science 313, 1027 (2006).

[3] H. Kesten, The work of Stanislav Smirnov., Laudations for Fields medal at ICM 2010 (2010).

[4] I. Rushkin, P. Oikonomou, L. P. Kadanoff, and I. A. Gruzberg, J. Stat. Mech. , P01001 (2006).

[5] P. Oikonomou, I. Rushkin, I. A. Gruzberg, and L. P. Kadanoff, J. Stat. Mech., P01019 (2008).

[6] D. Zhan, Probab. Theory Related Fields 129, 340 (2004).

[7] M. N. Najafi, S. Moghimi-Araghi, and S. Rouhani, J. Phys. A: Math. Theor. 45, 095001 (2012).

[8] S. Smirnov, C. R. Acad. Sci. Sér. I Math. 333, 239 (2001).

[9] G. F. Lawler, O. Schramm, and W. Werner, Proc. Sympos. Pure Math. 72, 339 (2002).

[10] W. Kager and B. Nienhuis, J. Stat. Phys. 115, 1149 (2004).

[11] J. Cardy, Ann. Phys. 318, 81 (2005).

[12] G. Lawler, O. Schramm, and W. Werner, in Selected Works of Oded Schramm, Selected Works in Probability and Statistics, edited by I. Benjamini and O. Häggström (Springer New York, 2011) pp. 931-987.

[13] E. Daryaei, N. A. M. Araújo, K. J. Schrenk, S. Rouhani, and H. J. Herrmann, Phys. Rev. Lett. 109, 218701 (2012).

[14] N. Posé, K. J. Schrenk, N. A. M. Araújo, and H. J. Herrmann, Sci. Rep. 4, 5495 (2014).

[15] D. Stauffer and A. Aharony, Introduction to percolation theory (CRC press, 1994).

[16] I. Dayan, J. F. Gouyet, and S. Havlin, J. Phys. A: Math. Gen. 24, L287 (1991).

[17] H. Hinrichsen, Adv. Phys. 49, 815 (2000).

[18] T. Kennedy, J. Stat. Phys. 131, 803 (2008).

[19] K. Löwner, Math. Ann. 89, 103 (1923). 

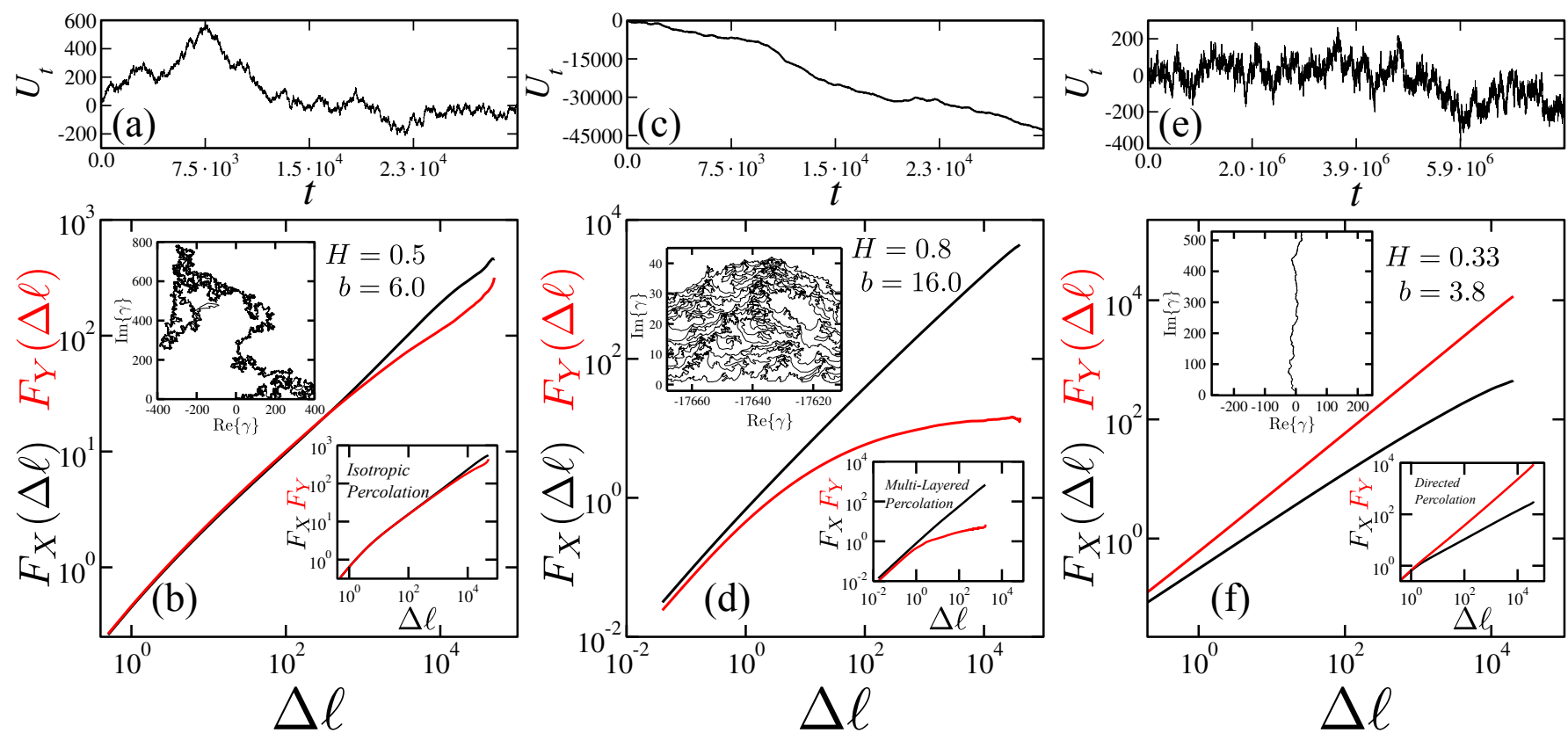

FIG. 4. Root mean squared estimations of the displacements in the $X$ and $Y$-directions of SLE traces driven by long-range power-law correlated time series (fractional Brownian motion). In (a), (c) and (e) we show typical realizations of uncorrelated, correlated and anti-correlated driving functions, respectively. The simulation parameters $\left(H, b\right.$ and $\left.t_{f}\right)$ were chosen based on the results shown in Fig. 3 (see Table \for the numerical values). Good agreement is observed between the uncorrelated result (b) and isotropic percolation (inset on the bottom), as it is expected. The correlated trails (d) are also compatible with multi-layered percolation (inset on the bottom). In the anti-correlated case (f), the same kind of anisotropy present in the directed percolation is observed (inset on the bottom). These results support our hypothesis that long-term correlations in the driving functions, i.e., the presence of anomalous diffusion, are responsible for the anisotropic behavior of the traces. The insets on the top of (b), (d) and (f) show examples of the traces generated from the simulations with the corresponding driving function shown in (a), (c) and (e), respectively.

[20] G. F. Lawler, Conformally invariant processes in the plane, Mathematical Surveys and Monographs, Vol. 114 (American Mathematical Society, 2005).

[21] V. Beffara, Ann. Probab. 36, 1421 (2008).

[22] S. Smirnov, in Proceedings of the International Congress of Mathematicians Madrid (European Mathematical Society, 2006) pp. 1421-1451.

[23] D. Bernard, P. Le Doussal, and A. A. Middleton, Phys. Rev. B 76, 020403(R) (2007).

[24] E. Bogomolny, R. Dubertrand, and C. Schmit, J. Phys. A: Math. Theor. 40, 381 (2007).

[25] A. Gamsa and J. Cardy, J. Stat. Mech. , P08020 (2007).

[26] R. O. Bauer, Ann. Fac. Sci. Toulouse Math. 12, 433 (2003).

[27] T. Kennedy, J. Stat. Phys. 128, 1125 (2007).
[28] J. Felsenstein, Evolution 39, 783 (1985).

[29] F. Camia and C. M. Newman, Commun. Math. Phys. 268, 1 (2006).

[30] R. M. Ziff, P. T. Cummings, and G. Stells, J. Phys. A: Math. Gen. 17, 3009 (1984).

[31] E. J. R. Parteli, L. R. Silva, and J. S. Andrade, J. Stat. Mech., P03026 (2010).

[32] R. M. Ziff, Phys. Rev. Lett. 56, 545 (1986).

[33] I. Jensen, J. Phys. A: Math. Gen. 32, 5233 (1999).

[34] B. B. Mandelbrot and J. W. V. Ness, SIAM Rev. 10, pp. 422 (1968).

[35] R. B. Davies and D. S. Harte, Biometrika 74, 95 (1987).

[36] C.-K. Peng, J. Mietus, J. M. Hausdorff, S. Havlin, H. E. Stanley, and A. L. Goldberger, Phys. Rev. Lett. 70, 1343 (1993). 